\title{
Hypothyroid myopathy with periodic paralysis as the main symptom: a case report and literature review
}

\author{
Ning Lin, Jiajun Wang, Wuping Liao, Ying Wen \\ Department of Chinese Medicine, Guangdong Second Provincial General Hospital, Guangzhou, China \\ Correspondence to: Ning Lin. Department of Chinese Medicine, Guangdong Second Provincial General Hospital, Guangzhou 510317, China. \\ Email: 740545648@qq.com.
}

\begin{abstract}
Hypothyroid myopathy is a skeletal muscle disease caused by hypothyroidism. However, patients with hypothyroidism are often misdiagnosed as polymyositis if they do not have a clear history of thyroid gland or obvious hypometabolic symptoms, but with myasthenia and myalgia as the main symptoms or the first symptoms. Moreover, hypothyroid myopathy with periodic paralysis as the first symptom is rare in clinic. In this study, we summarized the clinical data of 1 case of hypothyroid myopathy with periodic paralysis as the first symptom in our clinical diagnosis and treatment. A 27 -year-old male patient with recurrent periodic paralysis was found with hypothyroidism during a most recent attack of myasthenia and was diagnosed with hypothyroid myopathy, which was relieved after oral administration of levothyroxine. We also found 13 similar cases reported internationally, and summarized their clinical characteristics, diagnosis, and treatment methods to provide reference for the clinical diagnosis and treatment of such cases. In general, periodic paralysis may be the main symptom or even the first symptom of hypothyroid myopathy, which is easy to be confused with renal tubular acidosis (RTA) or other autoimmune diseases. The diagnosis is mainly based on the detection of thyroid function and thyroid autoantibodies. Timely supplement of thyroxine and correction of electrolyte disorders are the key to treatment.
\end{abstract}

Keywords: Hypothyroid myopathy; periodic paralysis; diagnosis; case report

Submitted Jul 17, 2020. Accepted for publication Sep 04, 2020.

doi: 10.21037/apm-20-1578

View this article at: http://dx.doi.org/10.21037/apm-20-1578

\section{Introduction}

Periodic paralysis is a type of myopathy related to potassium ion $(\mathrm{K}+)$ metabolism, which is clinically characterized by recurrent myasthenia with changes in serum potassium ion levels (1). Hypokalemic periodic paralysis (HOPP) is the most common type of periodic paralysis, with an incidence of about 1 in 100,000, and is more common in Asian men. HOPP can be divided into two types: primary (familial or idiopathic) HOPP and secondary (acquired) HOPP (2). Primary HOPP is caused by the intracellular shift of $\mathrm{K}+$ due to potassium channel gene mutations. Secondary HOPP is caused by a variety of etiologies; one of the most common causes is thyrotoxicosis, which is characterized by increased activity of the sodium-potassium pump caused by excessive thyroid hormones, leading to intracellular potassium shift (3). Hypothyroid myopathy (HM) is a skeletal muscle disease caused by hypothyroidism. About $30 \%$ to $80 \%$ of patients with hypothyroidism develop myopathy whose symptoms include myasthenia, muscle spasm after exercise, myalgia, and muscle stiffness (4). The symptoms of HM are usually mild, but tend to become more pronounced in untreated severe hypothyroidism (5). The skeletal muscle damage caused by hypothyroid myopathy is due to Glucosaminoglycan deposition in type II fast muscle fibers and type I slow muscle fibers, weakened contractility of actin-myosin unit, decreased myosin ATPase activity and slowed ATP turnover (6). The literature about neuromuscular complications in hypothyroidism is mostly based on retrospective analysis. In addition, the risk of hypothyroidism is higher in patients with myotonic dystrophy (7). Therefore, 
the true prevalence of neuromuscular complications during hypothyroidism is not easy to determine and may be underestimated. So, it is not difficult to diagnose hypothyroid myopathy if the patients have a clear history of hypothyroidism (primary hypothyroidism, secondary hypopituitarism hypothyroidism, autoimmune thyroiditis, post-thyroidectomy status, antithyroid drug prescription, etc.), or muscle lesions diagnosed according to the typical clinical symptoms of hypothyroidism (low basal metabolism syndrome, myxedema face, cardiovascular system, digestive system changes, etc.) (8). However, it is easy to misdiagnose hypothyroidism patients if they have no clear history of thyroid disease or no significant hypometabolism symptoms, but do have myasthenia and myalgia as the main symptoms or the first symptom (9). The condition most commonly confused with hypothyroid myopathy in clinic is polymyositis (10). However, hypothyroid myopathy with periodic paralysis as the first symptom is rare in clinic, and is mostly only known through case reports. This study summarized the clinical data of 1 case of hypothyroid myopathy with periodic paralysis as the first symptom in our clinical diagnosis and treatment. In the study, we also found 13 similar cases reported internationally, and summarized their clinical characteristics, diagnosis, and treatment methods to provide reference for the clinical diagnosis and treatment of such cases. We present the following article in accordance with the CARE reporting checklist (available at http://dx.doi.org/10.21037/apm-20-1578).

\section{Case presentation}

The patient was a 27-year-old male from Tianmen City, Hubei Province, and was admitted on September 23, 2017. Beginning 8 years prior, the patient had lower limb weakness and difficulty in walking. The examination in another hospital found low serum potassium, so the patient was a given potassium supplement for treatment with the symptoms being relieved after rest. On September 21, 2017, after exerting himself, the patient relapsed with lower limbs weakness and difficulty in walking but without loss of energy, fear of cold, lethargy, poor appetite, constipation, weight gain, or other symptoms. Consequently, the patient visited our emergency department for treatment. The examination results were as follows: serum potassium, $3.2 \mathrm{mmol} / \mathrm{L}$; muscle enzyme, serum free triiodothyronine (FT3), free thyroxine (FT4), and thyroid stimulating hormone (TSH) were normal. The symptoms were not significantly relieved after intravenous drip of potassium aspartate and oral administration of potassium chloride solution. On September 22, the serum potassium was $2.98 \mathrm{mmol} / \mathrm{L}$, and intravenous and oral potassium supplement was continued. On the night of September 22, the serum potassium was $3.64 \mathrm{mmol} / \mathrm{L}$, and the patient still felt weakness of the lower limbs, and so was admitted with periodic paralysis. The patient was previously healthy and denied any history of hypertension, thyroid dysfunction, or other diseases. His vital signs were normal: body temperature, $36.2{ }^{\circ} \mathrm{C}$; pulse, $72 / \mathrm{min}$; blood pressure $120 / 84, \mathrm{mmHg}$. The patient had no obvious thyroid enlargement, and no obvious abnormalities in the heart, lung, or abdomen. His muscle strength and upper limb muscle tension were generally normal, and he exhibited no obvious muscle atrophy or hypertrophy of the lower limbs, and no muscle tenderness. The muscle strength was grade 4, muscle tension decreased, and knee reflex and Achilles tendon reflex had weakened. Laboratory results during hospitalization, including blood biochemistry, urine biochemistry, and blood gas analysis, are shown in Table 1. Blood cortisol, aldosterone, and other examinations showed no abnormality. Electrocardiogram (ECG) revealed sinus rhythm and ST segment elevation. Chest X-ray showed no obvious abnormality. Abdominal color Doppler ultrasound showed fatty liver, with both kidneys and urinary bladder showing no obvious abnormality. Electromyography (EMG) showed no definite neurogenic or myogenic injury. The patient was diagnosed with HOPP, and continued oral and intravenous potassium supplement. Serum potassium on multiple retests was generally normal, while creatine kinase (CK) on multiple retests was elevated (Table 1). After 5 days, the subjective symptoms of the patient had improved with normal serum potassium. He refused further diagnosis and treatment due to personal reasons and was discharged.

On July 31, 2018, the patient again experienced weakness with pain in the lower limbs after exertion. Outpatient blood biochemistry and thyroid function detection are shown in Table 1. Liver enzymes were elevated: alanine aminotransferase (ALT), $81 \mathrm{U} / \mathrm{L}$ (reference value 0-50 U/L); aspartate transaminase (AST), $67 \mathrm{U} / \mathrm{L}$ (reference value 0-50 U/L). Twelve items of anti-ENA antibody were all negative. Thyroid color Doppler ultrasound showed diffused thyroid enlargement and abundant blood supply. EMG revealed no clear neurogenic or myogenic injury. According to these indicators, the patient was diagnosed with autoimmune thyroiditis complicated with hypothyroid myopathy, and was given levothyroxine sodium tablets $25 \mu \mathrm{g}$, 1 tablet/day orally. After 4 weeks, the patient indicated by 
Table 1 Laboratory results in the patient with hypothyroid myopathy with hypokalemic periodic paralysis as the first symptom

\begin{tabular}{|c|c|c|c|c|c|c|c|c|c|c|c|}
\hline Date & $\begin{array}{c}\mathrm{Na}+ \\
(\mathrm{mmol} / \mathrm{L})\end{array}$ & $\begin{array}{c}\mathrm{K}+ \\
(\mathrm{mmol} / \mathrm{L})\end{array}$ & $\begin{array}{c}\mathrm{CL}- \\
(\mathrm{mmol} / \mathrm{L})\end{array}$ & $\begin{array}{c}\text { CK } \\
(\mathrm{U} / \mathrm{L})\end{array}$ & $\begin{array}{c}\text { Urine K+ } \\
(\mathrm{mmol} / 24 \mathrm{~h})\end{array}$ & $\begin{array}{c}\text { Urine } \\
\mathrm{PH}\end{array}$ & Blood PH & $\begin{array}{l}\text { AnGap } \\
(\mathrm{mmol} / \mathrm{L})\end{array}$ & $\begin{array}{c}\text { TSH } \\
(\mathrm{ulU} / \mathrm{L})\end{array}$ & $\begin{array}{c}\text { FT4 } \\
(\mathrm{pmol} / \mathrm{L})\end{array}$ & $\begin{array}{l}\text { aTPO } \\
(\mathrm{U} / \mathrm{mL})\end{array}$ \\
\hline $2017-9-21$ & 142 & 3.2 & 96 & 164 & & & & & 5.4 & 15.08 & \\
\hline $2017-9-22$ & 141 & 2.98 & 100 & & & & & & & & \\
\hline $2017-9-23$ & 137 & 4.27 & 98.2 & 619 & & 8 & 7.3 & 20 & & & \\
\hline Reference value & $137-147$ & $3.5-5.3$ & $99.0-110.0$ & $26-218$ & & $5.0-8.0$ & $7.35-7.45$ & $8-16$ & $0.35-5.5$ & $11-23$ & $0-60$ \\
\hline
\end{tabular}

$\mathrm{CK}$, creatine kinase; TSH, thyroid stimulating hormone; FT4, free thyroxine.

Table 2 Clinical data of 5 previously healthy cases of hypothyroid myopathy with periodic paralysis as the first symptom

\begin{tabular}{|c|c|c|c|c|c|c|c|c|}
\hline Case & Gender & $\begin{array}{l}\text { Age } \\
\text { (year) }\end{array}$ & $\mathrm{K}+$ & $\mathrm{CK}$ & FT4 & TSH & $\begin{array}{l}\text { TPOAb } \\
(\mathrm{IU} / \mathrm{mL})\end{array}$ & Others \\
\hline 1. China (11) & Female & 36 & $3.37 \mathrm{mmol} / \mathrm{L}$ & $380 \mathrm{U} / \mathrm{L}$ & $0.32 \mu \mathrm{g} / \mathrm{dL}$ & $288.8 \mathrm{IU} / \mathrm{mL}$ & $>1,000$ & 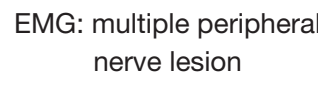 \\
\hline 2. India (12) & Female & 38 & $1.8 \mathrm{mmol} / \mathrm{L}$ & $241 \mathrm{U} / \mathrm{L}$ & - & $>100 \mathrm{mlU} / \mathrm{L}$ & 287 & \\
\hline 3. UK (13) & Female & 8 & Normal & Normal & $\begin{array}{l}11.1(8.5-24) \\
\mathrm{pmol} / \mathrm{L}\end{array}$ & $\begin{array}{c}10.9(0.35-4) \\
\mathrm{mU} / \mathrm{mL}\end{array}$ & 132 & \\
\hline 4. India (14) & Female & 30 & $\begin{array}{l}\text { Fluctuation } \\
1.6-3.2 \mathrm{meq} / \mathrm{L}\end{array}$ & $1,067 \mathrm{mg} / \mathrm{dL}$ & $\begin{array}{l}\text { Low level, no } \\
\text { specific values }\end{array}$ & $>100$ & 386.4 & EMG: normal \\
\hline 5. Sri Lanka (15) & Male & 30 & $2.2 \mathrm{mmol} / \mathrm{L}$ & $\begin{array}{c}234(24-195) \\
\mu / L\end{array}$ & Normal & $\begin{array}{c}16.5(0.3-4.2) \\
\mathrm{mU} / \mathrm{L}\end{array}$ & 324 & $\begin{array}{l}\text { EMG: multiple fibrous } \\
\text { myoclonus; Muscle } \\
\text { biopsy: normal }\end{array}$ \\
\hline
\end{tabular}

-, the results not reported; (), a range of reference values. CK, creatine kinase; TSH, thyroid stimulating hormone; FT4, free thyroxine; EMG, electromyography.

telephone follow-up that the weakness and myalgia of the lower limbs had disappeared.

We searched all relevant literature and found 13 cases with complete clinical diagnosis and treatment reports of hypothyroidism with periodic paralysis (Table 2). Of these, 5 cases with hypothyroid myopathy were previously relatively healthy without history of thyroid diseases or other underlying diseases. Periodic paralysis was their first symptom, and the course of periodic paralysis ranged from 2 weeks to 2 years. The main symptom was limb weakness, especially lower limb weakness, without respiratory muscle or sphincter involvement. The other 8 cases of hypothyroid myopathy with periodic paralysis were complicated with other clinical conditions (Table 3). The treatment of all cases is summarized in the discussion section. All procedures performed in studies involving human participants were in accordance with the ethical standards of the institutional and/or national research committee(s) and with the Helsinki Declaration (as revised in 2013). Written informed consent was obtained from the patient.

\section{Discussion}

We found a total of 14 relevant cases: 13 cases from literature review and 1 case from our study. Eleven cases (78.6\%) were from Asian countries, of which 6 (42.9\%) were from the South Asian countries of India and Sri Lanka, which is consistent with the epidemiological characteristics of sporadic HOPP being more common in Asian populations (23). However, in this study, the femaleto-male ratio was $10: 4$ (71.43\% in females and $28.57 \%$ in males), which is the opposite trend to the gender difference reported in the incidence of primary HOPP (about 38\% in females and $62 \%$ in males) (24). This may be due to 
Table 3 Clinical data of 8 cases of hypothyroid myopathy with periodic paralysis complicated with other clinical conditions

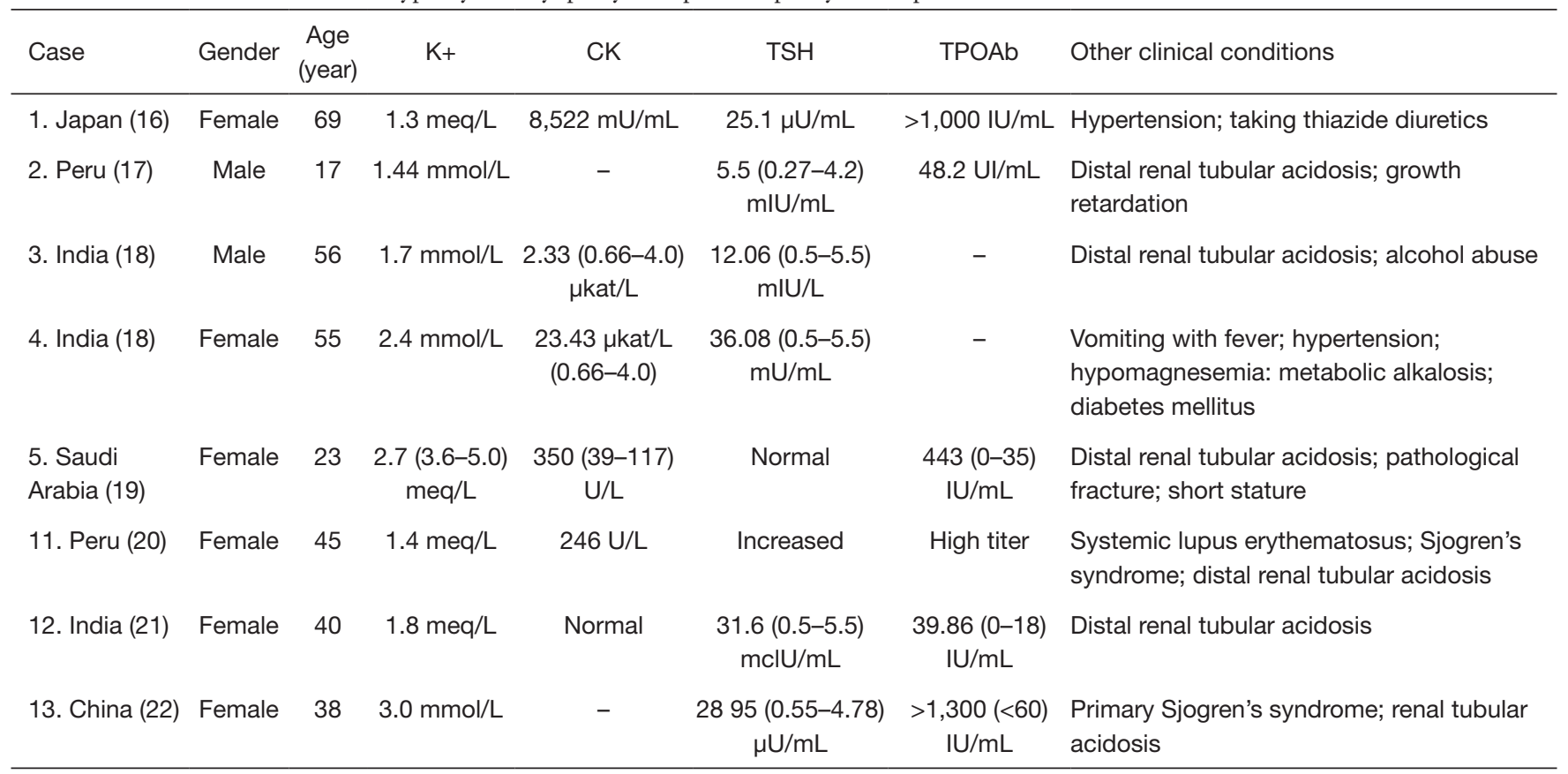

-, the results not reported; (), a range of reference values; $\mathrm{CK}$, creatine kinase; TSH, thyroid stimulating hormone.

the fact that primary diseases such as chronic lymphocytic thyroiditis and hypothyroidism have a higher incidence in the female population.

Renal tubular acidosis (RTA) is a chronic metabolic acidosis caused by distal renal tubular secretion of hydrogen ions and/or proximal bicarbonate reabsorption disorder, and is often accompanied by hypokalemia, and disorders of calcium and phosphate metabolism. It is also a common cause of HOPP. RTA is also common in autoimmune diseases such as Sjogren's syndrome, rheumatoid arthritis, systemic lupus erythematosus (SLE), and chronic lymphocytic thyroiditis. Chronic lymphatic thyroiditis, in turn, is the most common cause of hypothyroidism, which explains why as many as 6 of 14 hypothyroidism cases $(42.9 \%)$ had RTA. In addition to cases 11 and 13 , which were definitely diagnosed with Sjogren's syndrome, RTA in other cases was likely due to chronic lymphocytic thyroiditis $(20,22)$. Renal secretion of $\mathrm{H}+$ depends on the transport of distal convoluted tubule cells through the hydrogenpotassium ATP pump. In the state of hypothyroidism, the number and function of the hydrogen-potassium ATP pump are decreased, and there is a higher risk of acidosis and hypokalemia (25). HOPP is more likely to occur when combined with other factors affecting acid-base balance and water-electrolyte balance, such as taking diuretics, alcohol abuse, or diabetes $(16,18)$. RTA is diagnosed based on clinical symptoms and some auxiliary examinations. Clinical symptoms include hyperchloremic metabolic acidosis with normal anion gap, urine $\mathrm{PH}$ persistently $>5.5$, susceptibility to renal calculi, while auxiliary examinations include sodium chloride load test and furosemide test. In September 2017, during the onset of periodic paralysis, our patient experienced hypokalemia with acidosis (arterial blood $\mathrm{PH}$ 7.30), and alkaline urine (urine $\mathrm{PH}$ 8.0). The possibility of RTA was considered, but it was not confirmed because of the normal blood chloride level and increased anion gap, and the patient refused further examination.

Clear CK elevation was present in 9 of 14 patients $(64.3 \%)$, suggesting that it is a common symptom of hypothyroid myopathy. As the muscle weakness in hypothyroid myopathy mainly involves the pectoral girdle, pelvic girdle, and proximal limb skeletal muscles, which are similar to the sites involved in polymyositis, these patients are easily misdiagnosed as polymyositis. Because patients with polymyositis often present (20-40\%) with positive serum antinuclear antibodies (ANA), the detection of the ANA antibody profile is of limited value in differentiating hypothyroid myopathy from SLE and Sjogren's syndrome. EMG and muscle biopsy can provide key information for differential diagnosis. EMG shows myogenic injury 
in $70-90 \%$ of polymyositis cases, while EMG shows no specificity in hypothyroid myopathy, which can be neurogenic, myotonic, or normal. The typical symptoms of muscle biopsy in polymyositis indicate muscle fiber necrosis, inflammatory cell infiltration, and perifascicular muscle atrophy, while muscle biopsy in hypothyroid myopathy is mostly normal or shows slight uncharacteristic muscle pathological changes. However, EMG and muscle biopsy are difficult to use as routine examinations in clinic due to the limitation of objective conditions. Therefore, thyroid function examination and thyroid autoantibody examination are important for the diagnosis of patients with clinical symptoms of unexplained muscle weakness, hypokalemia, and elevated muscle enzymes. For patients with recurrent periodic paralysis, a single normal result in a thyroid function test cannot rule out the possibility of hypothyroid myopathy (as the case reported here). If the patient experiences recurrent muscle weakness, thyroid function should be reexamined in time.

In terms of treatment, 13 of 14 patients were treated with a potassium supplement (intravenous or oral). In case 3 (13), the patient had normal serum potassium at the onset of periodic paralysis, and limb weakness did not recur after thyroxine supplement. In the 13 patients with hypokalemia, the time for serum potassium to return to normal levels after potassium supplementation varied from hours to weeks, with 3-7 days being the most common duration. In a few cases $(18,20)$, serum potassium levels decreased in the initial phase of potassium supplement. In this condition, patients required more potassium supplement, and hypokalemia was relieved even a few hours after receiving continuous intravenous pumping of potassium (20). A total of 13 patients were treated with exogenous thyroxine supplement at a dose of $25-150 \mu \mathrm{g} /$ day, and thyroid function spontaneously returned to normal in case 6 (16) at 36 days after onset (without thyroxine supplementation). Of note, in some cases, after thyroxine therapy was started, hypokalemia could not be quickly relieved $(12,14)$, and serum potassium levels returned to normal after continued potassium supplement for 4 weeks after starting oral levothyroxine. In case 13, longterm oral potassium citrate was still required after discharge. The reason for this "delayed" correction of hypokalemia may be related to the diuretic effect and increased potassium excretion during the initial phase of thyroxine administration (15). In addition to potassium and/ or thyroxine supplement, patients with other clinical conditions (Table 3) were also given alkali, magnesium, antihypertensive, hypoglycemic, immunosuppressive, anti- osteoporosis, and other treatments according to the primary disease type. All patient symptoms were controlled after timely treatment. Among these patients, case 7 stopped taking levothyroxine without authorization 3 weeks after initial treatment, and developed more severe muscle weakness with hypokalemia 1 week after drug withdrawal. The patient was readmitted for potassium, alkali, and thyroxine supplementation, and the symptoms were relieved (17).

In summary, hypothyroid myopathy characterized by periodic paralysis is uncommon in clinic, but periodic paralysis has the potential to be the first symptom of hypothyroid myopathy. For patients with recurrent skeletal muscle flaccid paralysis, a comprehensive thyroid disease laboratory examination [thyroid function and antibodies like autoantibodies to thyroid peroxidase (TPOAb)] is recommended to exclude thyroid dysfunction (hyperthyroidism or hypothyroidism), regardless of serum potassium level abnormalities or obvious basic metabolic abnormalities. For patients of HOPP with RTA, chronic lymphocytic thyroiditis needs to be screened for, along with connective tissue diseases, such as Sjogren's syndrome and SLE. Correction of abnormal serum potassium level and supplementation of an appropriate amount of thyroxine can effectively control periodic paralysis caused by hypothyroidism. However, it should be noted that clinical conditions such as the diuretic effects of thyroxine supplementation and combined acidosis may lead to a slow increase of serum potassium in patients with hypokalemia. Thus the route, dose, and course of potassium supplementation may vary from person to person.

\section{Acknowledgments}

Funding: None.

\section{Footnote}

Reporting Checklist: The authors have completed the CARE reporting checklist. Available at http://dx.doi.org/10.21037/ apm-20-1578

Conflicts of Interest: All authors have completed the ICMJE uniform disclosure form (available at http://dx.doi. org/10.21037/apm-20-1578). The authors have no conflicts of interest to declare.

Ethical Statement: The authors are accountable for all 
aspects of the work in ensuring that questions related to the accuracy or integrity of any part of the work are appropriately investigated and resolved. All procedures performed in studies involving human participants were in accordance with the ethical standards of the institutional and/or national research committee(s) and with the Helsinki Declaration (as revised in 2013). Written informed consent was obtained from the patient.

Open Access Statement: This is an Open Access article distributed in accordance with the Creative Commons Attribution-NonCommercial-NoDerivs 4.0 International License (CC BY-NC-ND 4.0), which permits the noncommercial replication and distribution of the article with the strict proviso that no changes or edits are made and the original work is properly cited (including links to both the formal publication through the relevant DOI and the license). See: https://creativecommons.org/licenses/by-nc-nd/4.0/.

\section{References}

1. Akaba Y, Takahashi S, Sasaki Y, et al. Successful treatment of normokalemic periodic paralysis with hydrochlorothiazide. Brain Dev 2018;40:833-6.

2. Weber F, Lehmann-Horn F. Hypokalemic periodic paralysis. In: Adam MP, Ardinger HH, Pagon RA, et al., editors. GeneReviews. Seattle (WA): University of Washington, Seattle, 2018:1993-2019.

3. Nicoletti T, Modoni A, Silvestri G. Secondary hypokalemic periodic paralysis as a rare clinical presentation of Conn syndrome. Clin Neurophysiol 2018;129:2505-6.

4. Lee KW, Kim SH, Kim KJ, et al. A Rare Manifestation of Hypothyroid Myopathy: Hoffmann's Syndrome. Endocrinol Metab (Seoul) 2015;30:626-30.

5. Ciompi ML, Zuccotti M, Bazzichi L, Puccetti L. Polymyositis-like syndrome in hypothyroidism: report of two cases. Thyroidology 1994;6:33-6.

6. Sindoni A, Rodolico C, Pappalardo MA et al. Hypothyroid myopathy: A peculiar clinical presentation of thyroid failure. Review of the literature. Rev Endocr Metab Disord 2016;17:499-519.

7. Johnson NE, Abbott D, Cannon-Albright LA. Relative risks for comorbidities associated with myotonic dystrophy: a populationbased analysis. Muscle Nerve 2015;52:659-61.

8. Aslam H, Sayeed MA, Qadeer R, et al. Hypothyroidism simulating as polymyositis. J Pak Med Assoc 2015;65:559-60.

9. Lee KW, Kim SH, Kim KJ, et al. A Rare Manifestation of Hypothyroid Myopathy: Hoffmann's Syndrome. Endocrinol Metab (Seoul) 2015;30:626-30.

10. Lin RT, Liu CK, Tai CT, et al. Hypothyroid myopathypathological and ultrastructural study. Kaohsiung J Med Sci 2000;16:68-75.

11. Jian H, Jingbing C. A case of hypothyroidism with initial symptom of myasthenia was reported. Chinese Journal of Practical Nervous Diseases 2013;16:106-7.

12. Chauhan P, Naxane A. Hypothyroidsm with Recurrent Hypokalemic Paralysis. Int J Sci Re 2017;6:2131-2.

13. Bandhakavi M. Periodic paralysis as an unusual presentation of autoimmune hypothyroidism with goiter. Clin Pediatr (Phila) 2009;48:677-8.

14. Sinha U, Sengupta N, Sinharay K, et al. Recurrent hypokalemic paralysis: An atypical presentation of hypothyroidism. Indian J Endocrinol Metab 2013;17:174-6.

15. Arambewela MH, Sumanathilaka MR, Pathirana KD, et al. A possible association of hypokalaemic periodic paralysis, autoimmune thyroiditis and neuromyotonia. Ceylon Med J 2013;58:175-6.

16. Kanemaru Y, Aizawa K, Kagami T, et al. A case of hypokalemic myopathy associated with transient hypothyroidism. Endocrinol Jpn 1984;31:809-14.

17. Meregildo-Rodríguez ED, Failoc-Rojas VE. Case Report: Recurrent hypokalemic periodic paralysis associated with distal renal tubular acidosis (type 1) and hypothyroidism secondary to Hashimoto's thyroiditis. F1000Res 2018;7:1154.

18. Kadeeja N, Senthilnathan N, Viswanathan S, et al. Sporadic hypothyroidism-related hypokalemic paralysis: Diagnosis in a resource-poor setting. J Family Med Prim Care 2017;6:862-4.

19. Basak RC, Sharkawi KM, Rahman MM, et al. Distal renal tubular acidosis, hypokalemic paralysis, nephrocalcinosis, primary hypothyroidism, growth retardation, osteomalacia and osteoporosis leading to pathological fracture: a case report. Oman Med J 2011;26:271-4.

20. Velarde-Mejía Y, Gamboa-Cárdenas R, Ugarte-Gil M, et al. Hypokalemic Paralysis: A Hidden Card of Several Autoimmune Diseases. Clin Med Insights Arthritis Musculoskelet Disord 2017;10:1179544117722763.

21. Naveen L, Malkarnekar S. Adult-Onset Distal Renal Tubular Acidosis with Hypokalemic Quadriparesis in a Patient with Autoimmune Hypothyroidism. J Integr Nephrol Androl 2014;1:82-4.

22. Hongmei $Z$, Weiwei $Z$, Xiaoyong Li, et al. Approach of the patient with hypokalemia caused Hashimoto's thyroiditis 
with primary Sjogren's syndromeby and renal tubular acidosis. Chin J Endocrinol Metab 2016;32:349-52.

23. Luft FC. Abrupt hypokalemia with paralysis from a clinician's perspective. J Mol Med (Berl) 2005;83:167-9.

24. Wang W, Jiang L, Ye L, et al. Mutation screening in Chinese hypokalemic periodic paralysis patients. Mol Genet Metab 2006;87:359-63.

Cite this article as: Lin N, Wang J, Liao W, Wen Y. Hypothyroid myopathy with periodic paralysis as the main symptom: a case report and literature review. Ann Palliat Med 2020;9(5):3698-3704. doi: 10.21037/apm-20-1578
25. Suzanne M, Martin D, Eddine TA, et al. Hypokalemia paralyzing revealing a rare association of autoimmune diseases: type 1 diabetes, thyroiditis and tubulopathy about a case. IOSR Journal of Pharmacy 2015;5:5-7.

(English Language Editor: J. Gray) 